\title{
Specialized Environment for Medical Radiological Image Visualization
}

\author{
Vincenzo Di Lecce and Andrea Guerriero \\ Dipartimento di Elettrotecnica ed Elettronica - Politecnico di Bari \\ Via Orabona n.4 - 70125 Bari - Italy - E-mail DILECCE@POLIBA.IT
}

\begin{abstract}
In recent years the digital techniques for medical pictures archival and communication systems (PACS) and teleradiology have been object of a number of studies.

Main investigated fields are: image detection, storage and distribution in order to substitute the ordinary paper and film based files, remote diagnosis, image-guided surgery and virtual reality. At present, digital image system are used as integration (quick look system) of conventional systems (film based) or for digital generated radiological imaging (i.e. ultrasound, computer tomography).

In this paper the limits of digital medical image systems, reduced resolution of the VDT, reduced contrast, large digital image file size, are discussed and a proposal for a specific environment is presented.
\end{abstract}

\section{Introduction}

Images of various kinds are increasingly important to medical diagnostic processes and serious problems are encountered in selecting the most appropriate imaging modalities, acquiring optimal quality images, and processing images to obtain highest quality information. Systems are being developed that help automate the processing and analysis of images and that help the synthesis and visualization of information from diverse sources.

Pictures have been traditionally taken with photographic film, which then has to be processed before images can be viewed. The development of digital camera now allows pictures to be taken and shown (printed or displayed) immediately. In medicine this can be an important advance, and, even though the quality of images is currently lower than that of photographic film, the huge advance of immediateness often far outweighs the slight loss in resolution. Digital techniques are now available with improved resolution, suitable to replace the standard film camera for taking routine clinical photographs as well as for use in specialized areas of medicine.

Photographic film has been used as the acquisition, storage and display medium for Xray image for the past 100 years. When ultrasound scanning was developed the images were displayed on a monitor. At present, with current expansion in the variety of digitally acquired images and the increasing capacity of digital storage systems, the possibility of dispensing without photographic film has been raised. Filmless imaging can lead to important saving in disposable resources and staff time and a major improvement in departmental efficiency.

In fact digital pictures must be acquired, stored, retrieved on side or in remote, without technical limits in size, resolution and number. The potential advantage of filmless imaging are "non film handling", "no storing and searching" for films and plates, "no sending" films for wards or clinics, image fast availability, telediagnostic. But in realistic systems (usable and cost effective systems) it is necessary to make a 
few compromises. They are: store only digital generated images as ultrasound, computer tomographic, magnetic resonance scan, hold images on line for a limited period only, compress data files with lossles technique, integration with films and plates. The main problems for a full digital system are:

- storage of a large amount of large image data files;

- network load and time expensive remote access;

- inadequacy of VDT based display systems for high resolution images.

\begin{tabular}{|l|c|}
\hline \multicolumn{1}{|c|}{ digital image } & size of image file \\
\hline ultrasound scan & $250 \mathrm{~kb}$ \\
\hline computer tomografy or magnetic resonance image & $1 \mathrm{Mb}$ \\
\hline digital mammography & $4 \mathrm{Mb}$ \\
\hline high resolution chest X-ray & $16 \mathrm{Mb}$ \\
\hline high resolution digitalization of a X-ray plat of angiography & $270 \mathrm{Mb}(12$ bit $)$ \\
\hline
\end{tabular}

Tab.1: File size of digital images

The inadequacy of VDT is not the only limit in X-ray displaying. Radiologists are used to have eight or more viewing boxes of films on display at once, often with a mixture of examinations of the same patient under review simultaneously. To reproduce this with large, high resolution, computer screens and associated software is very expensive. Most practical systems use two or more screens at each workstation, but the screen resolution (less than $100 \mathrm{dpi}$ ) is not comparable to film.

\section{Medical image size adjust}

In this chapter a comparative evaluation of reduction algorithms is presented in terms of computational complexity and information loss. One of the most confusing issues about images is how to measure the image quality. For resized images only subjective determination can be applied and these should be made by the end-user of the image under actual viewing condition (monitor contrast and size, image resolution) and environment (preparation and degree of attention of the observer, influence of the semantic interpretation of the image, reasoning ).

In the present case the original X-ray plate, of 11 "x 15 ", was acquired using a rotary scanner at $1200 \mathrm{dpi}$ (to resolve the small vessel around 1/25"). The picture of $13200 \times 18000$ pixels was reduced using the following algorithms:

- local algorithms:

undersampling;

weighted undersampling;

spectrum based algorithms : local Fast Fourier transform (LFFT); local Discrete Cosine transform (LDCT);

- global algorithms:

spectrum based algorithms : global FFT (GFFT);

$$
\text { global DCT (GDCT); }
$$

interpolation with low-pass filter in frequency domain; wavelet. 
The elements of these algorithms are in [1] for DCT based techniques, and in [2] for FFT and wavelet based techniques. The final size of the image, suitable for windows based displaying, is $512 \times 512$ pixels with a maximum linear reduction ratio about 20 . In this new size a small vessel of $1 / 25^{\prime}$ became of 2 pixel.
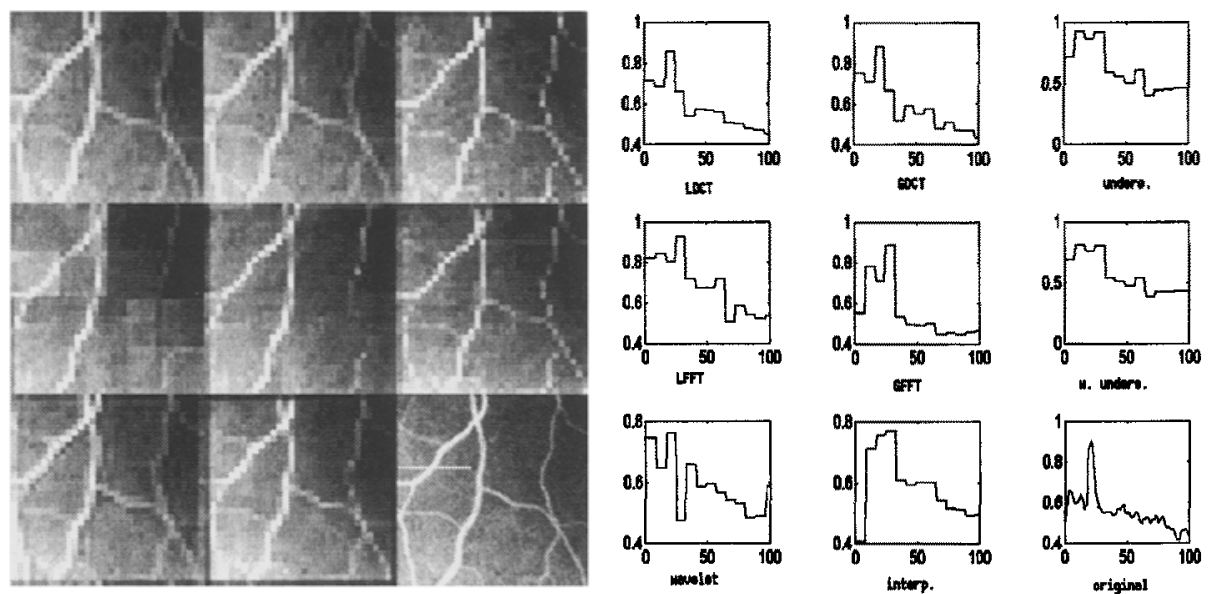

Fig. 1 : Effect of discussed algorithms on a X-ray image. Reduction ratio 10. From top-left: LDCT, GDCT, undersampling, LFFT, GFFT, weighted undersampling, wavelet, interpolation with low-pass filter and original image. The reduced images were restored in size, using linear interpolation, to evaluate the loss in vessel detectability. The images gray levels are normalized. The white row in the original image is the base for the plots on the right.

The reduced image can be used typically for a quick look (finalized to projection or frame selection). The confidence diagnosis requires a pre-defined image resolution. The image resolution depends on the smallest interesting feature to be detected, as discussed in the vessel case.

To improve the image quality, digital enhancement techniques can be used. Several techniques are based on template matching [3] but the reduction algorithms modify the expected template (as shown in the following). For this reasons the enhancement can be performed only on original image (or small window) or on reduced images characterized by large structures uncorrupted by the reduction (scoliosis evaluation).

In fig. 1 and tab. 2 the comparative evaluation of the discussed algorithms is presented. Fig. 3 show the image sections.

\section{Features extraction}

The characteristics of the image data, the nature of the particular application, the display medium and the properties of the human visual system determine the most appropriate enhancement processing method to convert the original image to a form more convenient for information extraction. 
All image enhancement techniques try to attenuate irrelevant features and at the same time to emphasize features of interest for the medical diagnosis protocol.

Typically, enhancement methods may be divided into:

- contrast and color enhancement (gray scale modification, pseudocolor and false color);

- edge enhancement;

- noise reduction.

The fundamental enhancement for $\mathrm{X}$-ray images is the contrast adjusting, to produce a picture that optimally uses the dynamic range of a display device.

For the human eye to see subtle changes in brightness, the contrast characteristics of an image must be adapted to the subjective brightness range.

A region which differs in luminance from its surrounding by less than $2 \%$ is indistinguishable to the human eye. The aim of contrast enhancement is to increase image contrast over this threshold.

The simplest method of increasing the contrast in an image with a reduced amplitude range, is the rescaling of each pixel (contrast stretching). There exist several way to scale pixel amplitude, the original image can be linearly mapped over its range, or the extreme amplitude values may be clipped to maximum and minimum limits (windowlevel transformation), or an absolute value transformation can be utilized for visualizing an image with negative value pixels.

\begin{tabular}{|l|c|}
\hline \multicolumn{1}{|c|}{ Algorithm } & operation count \\
\hline undesampling & $\left(N^{2}-N^{2} / r^{3}\right)$ \\
\hline weighted undesampling & $\left(N^{2}-N^{2} / r^{3}\right)$ \\
\hline local Fast Fourier Transform & $\left(N^{2} / r^{*}(1+1 / r)\right)$ \\
\hline local Discrete Cosine Transform & $O\left(N^{2}\left(\log _{2} 2 N-1 / r^{2}\right)\right)$ \\
\hline global Fast Fourier Transform & $O\left(N^{2}\left(\log _{2} N-1 / N^{2}\right)\right)$ \\
\hline global Discrete Cosine Transform & $O\left(N^{2}\left(\log _{2} N\right)\right)$ \\
\hline interpolation with low-pass filter & $O\left(N^{2}\right)$ \\
\hline wavelet & \\
\hline
\end{tabular}

Tab. 2: Computational load for different algorithm, $r$ represent the reduction ratio.

With these techniques the enhanced image may occupy only a portion of the dynamic range of the display, or may lie partly outside the dynamic range. To modify the image contrast utilizing the dynamic range of a display, nonlinear point transformation can be utilized. Square function, square root function, Gaussian error function, reverse and inverse function are same example of nonlinear point transformation which improve visual contrast [4]. 
Another important type of contrast enhancement is histogram modification, in which a gray scale transformation is used to give the picture a specified distribution of gray values. Two frequently used distributions approximate a normally distribute (Gaussian) or a flat (constant) histogram.

Histogram equalization ( flattening) produces pictures with higher contrast, because the points in the densely populated regions of the gray scale are forced to occupy a larger number of gray levels, resulting in these regions of gray scale being stretched.

If the histogram modification is obtained by a mapping function based on the histogram of pixels within a moving window on the image, we have an adaptive histogram modification. which produces good contrast enhancement in X-ray medical images [4]. Ideally, a histogram is generated in a window centered on each pixel of the image, equalized and used to calculate the new value for the pixel. This technique results computationally very intensive, so technique have been developed to interpolate between a reduced number of histogram equally spaced trough the image to obtain the equalized image [5]. Locally adaptive histogram equalization (LAHE) allows to enhance contrast in radiological image, which have a large global dynamic range, but small local feature gray level variations [6].

The optimal size of histogram area is difficult to determine and often varies from one area of the image to another.

A modified technique for contrast equalization is based on local DCT (LCE_DCT).

The parameters optimization for each image make arduous the use of these techniques in medical environment.

In radiological images higher spatial frequencies are weakened more than lower frequencies, due the low-pass characteristic of the $X$ ray system. Sharpening or crispening techniques are used to deblur the images emphasizing high spatial frequencies.

Blurring is an averaging or integration operation, therefore deblurring could be obtained by a differentiation operation. Simple differentiation operators are the gradient and the Laplacian. Other methods of edge enhancement are to subtract the Laplacian from the blurred image or high pass filtering, or unsharp masking, or statistical differencing the image [4]. Enhancement of fine details may also be obtained by homomorphic filtering based on the illumination - reflection model [7].

When an image is blurred and noisy, high pass filtering or differentiation cannot be used indiscriminately to sharpen it, since noise generally involve high rate of change of gray levels and hence high spatial frequencies. Therefore the noise should be reduced before edge sharpening.

$\mathrm{X}$ ray images are known to have signal dependent Poisson distributed noise that varies over different parts of the image depending on the intensity at each point. Several noise model have been derived to develop post processing algorithms to filter images corrupted by this type of noise [8-9].

\section{Graphical User Interface - Viewing Station}

In this section the main features of the proposed Graphical User Interface (GUI) are discussed. This GUI is devoted to radiological images for applications in medical telediagnostic, in particular for the viewing of high resolution digitized images with characteristics comparable to conventional radiography and for the improving of the image quality to allow the radiologist to make his diagnosis with more confidence. In 
fact to equalize the spatial resolution performance of conventional radiography, digitized images require pixel size smaller than $1 / 25$ " square, involving matrices too larger for the available display resolution; for this reason a resizing technique is needed. Moreover digital signal processing techniques can be applied in order to improve the image quality. Designing a GUI for radiological applications involves subjectiveness in medical diagnostic, therefore image processing techniques must be adapted to each image, and it depends on display device.
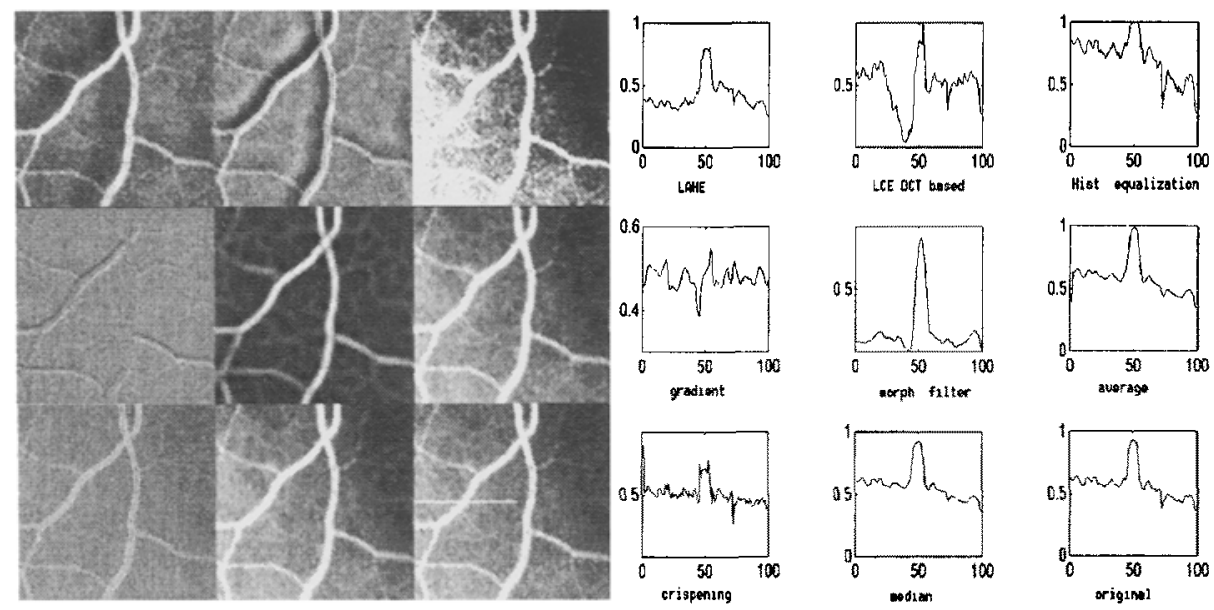

Fig. 4: Enhancement of X-ray image. From top-left: LAHE, LCE_DCT based, histogram equalization, gradient, morphological filter, average, crispening, median and original image. The white row in the original image is the base for the plots in right section

Moreover the original images must be preserved for different examinations and not permanently modified.

This GUI provides tools with the following characteristics:

- availability for processing of high-resolution original images: this means that all processing is carried out on not degraded images (reduced images);

- adaptability to specific image parameters, because the large variation in image features requires high flexibility on processing, even in images of the same kind;

- preprocessing capability on image windows, to allow user to test processing on a selected part of the image.

We consider :

- display tools ( resizing, cropping );

- enhancement tools (de-noising, contrast manipulation, edge crispening).

To view an image stored in the archive, the user, by a click on the "Open" item in the "File" pop-up menu, can choose the image to open. In the same menu the user finds the commands to save or print the processed image, and the commands to save or edit the log file that contains the list of commands executed to process the image. The enhanced images can be saved out of patient archive, in a temporary work area. Otherwise the commands sequence, utilized to produce the displayed image, is saved in the patient archives under a process ID. 
The GUI was developed in a windows environment. Every image is displayed in a $512 \times 512$ window, each window has an associate status area that contains the following information:

- the image reduction ratio;

- a graphical representation of the image portion displayed;

- a text list with the last 10 commands executed on the image.

To show high resolution images in $512 \times 512$ pixels windows, image size must be reduced.. As discussed in chapter II, several image resizing techniques exist, each one characterized by particular properties. Subsampling is the default resizing technique.

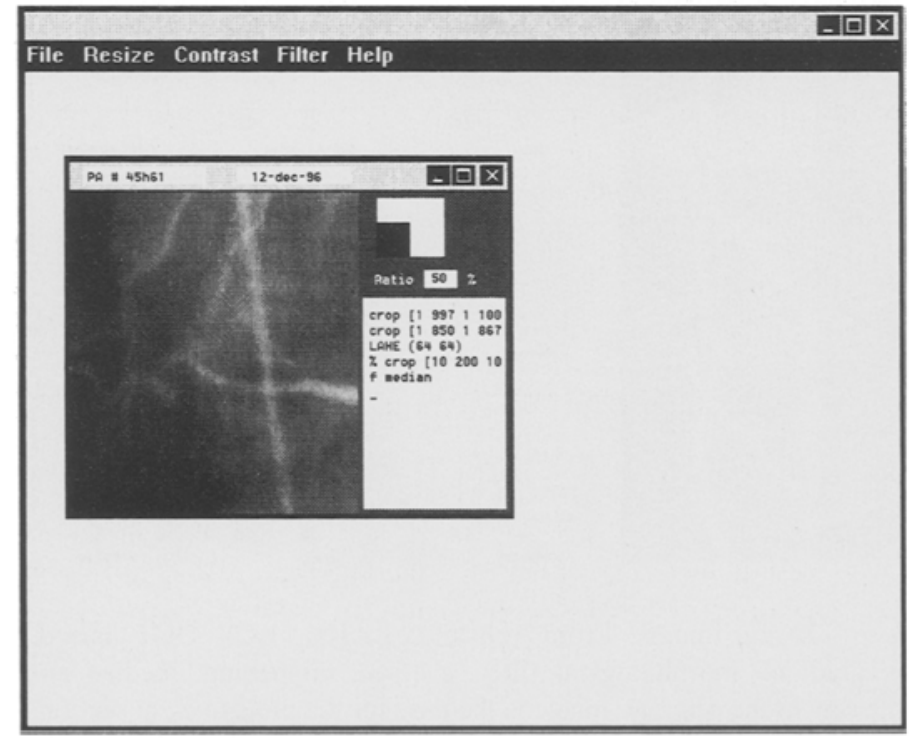

Fig 6: Hard copy of the proposed GUI prototype. The win-dow show a enhan-ced portion of an angiography. In the status area are dis-played the used command. The marker \% is generated by "undo" command.

Depending on the features of the processed image, and on the reduction ratio, the user can choose by the "Resize" pop-up menu the more convenient technique from a list. In this list the user can find also the crop operation.

To increase the image contrast the user can act on the "Contrast" pop up menu selecting the non adaptive or the adaptive histogram equalization. The window size for the local technique can be chosen by a slider.

By the "Filter" pop up menu it is possible to select a filter to emphasize feature of interest. High pass, median, pseudo median and unsharp masking are the available filters. More sophisticated filters, such as Wallis statistical differencing, Kalman or homomorphic [4], that require design and optimization can be imported.

The last menu provide the possibility to undo the last command, re-executing the log file except the last command, to execute the processing on the whole image or just to estimate the time required for the processing. Every time $\log$ file is executed, the command processor embedded in the GUI, optimizes the commands sequence to reduce the computation time. For example two or more consecutive crop operation or two consecutive filter operations, defined in the frequency domain, are substitute with the equivalent operation. 


\section{Conclusion}

In this paper starting from the analysis of physical limitation of VDT for medical applications, a specialized interface has been defined. The main features of the interface are:

- on line selectable resizing algorithms for displaying;

- availability of specific tools for image enhancement;

- processing tuning on a test window with log-file;

- log-file suitable for the GUI command processor;

- time-estimator included in the command processor;

- optimized command sequence obtained from the log file.

The reduction algorithms available are undersamplig (no computational load), wavelet (reduced computational load) and LDCT (high small features preservation). The effects of the reduction algorithms are shown in fig. 2 and 3.

The enhancement algorithms are "ready to use", without parameters optimization or specific design.

Optimized filters, for specific application, can be loaded in the environment.

At present we are working on a prototypal version of the proposed GUI for testing in a medical department.

The aim is in the integration of high-resolution X-ray image in PACS.

\section{Acknowledgements}

The authors would like to tank Dr. Donato Quagliara, Department of Cardiology, University of Bari, for providing the high-quality $\mathrm{X}$-ray angiography; Dr. Silvana Guerriero, Department of Ophthalmology, University of Bari, for providing retinal images; Dr. Rita Dario, Case di Cura Riunite-Bari, for the preliminary test of the GUI.

\section{Bibliography}

[1] B. Chitprasert, K.R. Rao : "Discrete Cosine Filtering", Proceedings of the 1990 IEEE International Conference on Acoustics, Speech and Signal Processing.

[2] G. Strang, T. Nguyen : "Wavelet and Filter Banks", Wellesley Cambridge Press 1996.

[3] S. Chaudhuri et al.: "Detection of blood vessels in retinal images using two-dimensional matched filters", IEEE trans. on medical imaging, Vol.8, no.3, september 1989.

[4] W.K. Pratt: "Digital Image Processing", John Wfley and Sons, U.K. 1978.

[5] S.M. Pizer et al., "Adaptive Histogram Equalization and its Varitions", Computer Vision, Graphics, and Image Processing, 39, 3, September 1987.

[6] W.M. Morrow et al., "Region Based Contrast Enhancement of Mammograms", IEEE Transaction on Medical Imaging, Vol. 11, No.3, September 1992.

[7] A.V Oppenheim, R.W. Schafer : Digital Signal Processing, Prentice Hall, Englewood Cliffs, N.J. 1975.

[8] C.M. Lo A.A. Sawchuk : "Nonlinear restoration of filtered images with Poisson noise", in Proc. SPIE vol. 207, 1979.

[9] W.A. Pearlman, W. Song : "A robust method for restoration of photon-limited, blurred images", in Proc. SPIE vol. 504, 1984. 